\title{
Using DLNA Cloud for Sharing Multimedia Contents beyond Home Networks
}

\author{
Van Dang Khoa Mai, Younghan Kim \\ School of Electronic Engineering, Soongsil University, Dongjak-gu, Seoul, 156-743, South Korea \\ mvdkhoa@dcn.ssu.ac.kr, younghak@ssu.ac.kr
}

\begin{abstract}
The recent emergence of Digital Living Network Alliance (DLNA) standard has made multimedia content sharing among devices in home networks easier than ever before. Several studies have focused on extending the operation of DLNA devices beyond the home networks based on devices called home gateways (HGs). However, these architectures just limit on sharing between two home networks. In order to connect with another home network or when the IP address of a HG is changed, they require the reconfiguration from users. This paper proposes a novel architecture in which a DLNA cloud (DC) plays a role in managing all of HGs. The HGs just need to be configured in the first time connecting to the DC. Sharing policy is built based on the users' relationship in a social network. In this architecture, DLNA users can easily share their content with their friends over the Internet. External multimedia content is transparently played in the home network as if it is located at user's HG. In addition, we also propose procedures for communication between the HG and the DC; as well as between the HG, the DC and the social network system (SNS).
\end{abstract}

Keywords —DLNA, home network, content sharing, n-screen, DLNA Cloud

\section{INTRODUCTION}

The DLNA [1] is becoming a popular standard for content sharing in home networks. However, DLNA standard cannot satisfy the demand of sharing contents among home networks over the Internet. Many previous studies extended the DLNA architecture to bring external contents into home networks. These architectures required the special device which is called HG. This device uses a public IP address to communicate with external world. Using such architectures, the users who use a dynamic public IP will face difficulties in configuration to communicate with each other.

The architectures in [2] and [3] just allow sharing between two home networks, and end users have to configure manually to establish the connection between two HGs. If end users want to connect with another home or the IP address of the HG changes, they have to reconfigure from the scratch.

Although the architecture in [4] allows many HGs to join the media cloud but not mention any specific mechanism for joining and sharing among them.

Authors of [5], [6], and [7] using P2P protocols to obtain contents from external networks to home networks. However, contents in P2P networks usually have different media formats from DLNA media formats. Therefore, those systems require a media conversion phase before being played in DLNA devices. The conversion phase results in increasing the total delay time of the system.

On the other hand, cloud computing is a tendency of the computer industry. Nowadays, most of big online services are deployed based on the cloud architecture. Consequently, end users benefit from using cloud services which is always available and up-to-date without technical understanding about the underlying infrastructure.

For those reasons, this paper proposes a novel architecture for sharing content between DLNA devices over the Internet. In this architecture, for keeping the designing philosophy of DLNA protocols which just limit in the home network, each home network still need a HG. However, two new components join our architecture. The first one is the DC. This component manages all DLNA HGs and maintains the sharing policy between them. Besides, the DC collects information of available contents in home networks and provides this information to other HGs. In this architecture, the HG just needs to be configured at the first time before using. The IP address changes of the HGs are automatically updated and managed by DC. The second new component is the SNS. This is an external existing component. We just obtain information from the existing relationships on social network infrastructures to build our sharing policy for our DC. When the relationships in the social network are updated, the sharing policy in the DC is updated too. In our architecture, the multimedia content in user's home network is shared automatically with their friends in the social network. However, a DLNA device in a certain home network sees all devices joined the same DC as the only device at its HG. Figure 1 describes the overall system architecture.

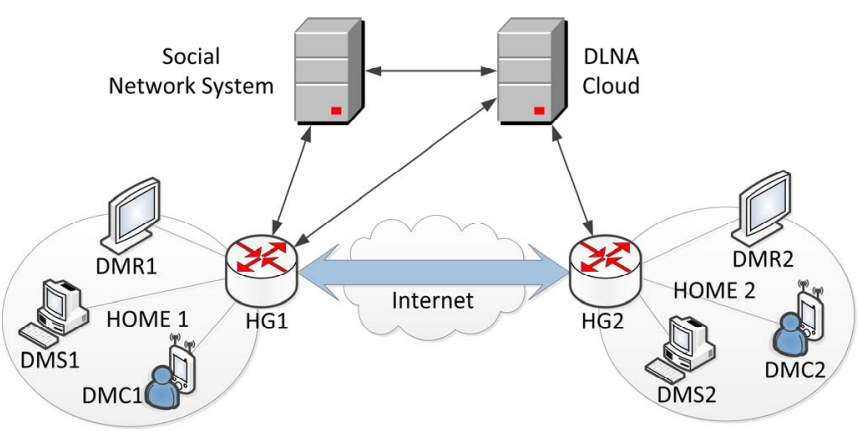

Figure 1. Overall system architecture 


\section{BACKGROUND}

\section{A. DLNA}

DLNA released guidelines for interoperability between media devices in home network environments. They include, but are not limited to, televisions, tablets, smartphones, laptops, printers and video recorders. The devices that follow these guidelines are certified by DLNA organization. In home network, these devices can easily get their IP address, discover, and share contents with each other.

DLNA Guidelines contain two main parts: ArchitectureProtocols and Media Formats. Electronics vendors use this information to build interoperable devices for home networks. Currently, DLNA guidelines have adopted UPnP Device Architecture 1.0 and UPnP AV Architecture for device discovery, control and media management, respectively.

According to the guidelines, devices in home networks are classified into three main categories: Digital Media Server (DMS), Digital Media Renderer (DMR), and Digital Media Controller (DMC). DMSs are devices which expose and distribute media content. Meanwhile, DMRs are devices which have the ability to render contents from DMSs and display it on their monitor. The last, DMCs are devices which control the cooperation between DMSs and DMRs. Additionally, DMCs have the ability to take basic operations remotely on DMRs such as: play, stop, pause, next or adjust the volume of DMRs.

\section{B. Cloud computing}

Cloud computing refers to both applications delivered as services over the Internet and the hardware and system software in the data centers that provide those services [8]. There are three cloud computing service models: Software as a Service (SaaS), Platform as a Service (PaaS), and Infrastructure as a Service (IaaS). Most of the current clouds are built on the top of modern data centers [9]. Figure 2 describes the hierarchical view of Cloud computing. IaaS provides for users or upper layer an abstract layer, which is independent of computing power, storage and network connectivity below. PaaS provides services for designing, developing, testing, deploying, and monitoring applications on the cloud. SaaS is used by users as a service on demand. End users can use the cloud-based software through a Web browser. They do not need to set up or maintain anything. The software is always available and up-to-date automatically.

\begin{tabular}{|c|}
\hline Software as a Service \\
\hline Platform as a Service \\
\hline Infrastructure as a Service \\
\hline Data Centers \\
\hline
\end{tabular}

Figure 2. Hierarchical view of cloud computing

Moreover, the services provided by the cloud can be accessed by users from anywhere at any time. User data can be stored and shared easily and safely via the Cloud regardless of any knowledge about the underlying infrastructure.

\section{III.PROPOSED SCHEME}

\section{A. Overall Architecture}

In our architecture, each home network has to equip a HG. In addition, each HG has two network interfaces: one network interface with a private IP address for connecting to the DLNA home network and another network interface with a public IP address for connecting to the Internet. These HGs are intermediate devices for communication between home networks and external networks. The DC manages all HGs which join to the system. All signaling messages are exchanged between the DC and the HG. However, data messages are exchanged directly between HGs without the presence of the DC.

Each HG must be specified the DC to connect to in its configuration. Besides, user also has to choose a SNS, gives necessary access information (username and password) and permissions for the DC to access this SNS to obtain user's information and social relationships.

With this architecture, when the IP address of a HG is changed, this $\mathrm{HG}$ will automatically register to the DC again without the intervention from user. The HG just needs the configuration from user only once at the initial phase of the system.

\section{B. Detail Procedures}

\section{1) Registration and Authentication Procedure}

Figure 3 shows the registration and authentication procedure. At the beginning, the $\mathrm{HG}$ sends the register message to the DC. The DC will redirect this request to the SNS login page. At this time, the user has to provide the login information to access to the SNS. This information can be remembered for later connections. If the login information is correct, the HG gets Access token from the SNS. Afterward, the HG forwards this token to the DC. By sending this token to the SNS, the DC can check whether the user is valid. If the user is valid, the DC can get the user ID and user's friend ID list.

Each time the HG connects to the DC, this procedure is repeated. The new relationships in the SNS, if any, are updated to the DC database. As the result, the sharing policy between HGs is updated too.

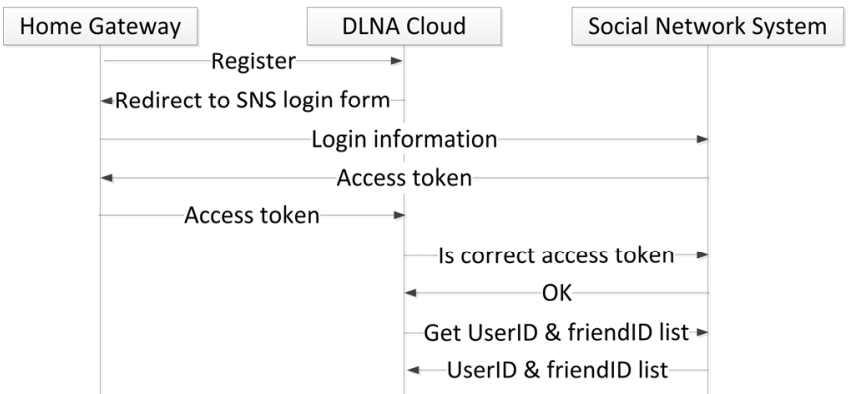

Figure 3. Registration and authentication procedure 


\section{2) Discovery and Service Description Procedure}

Figure 4 describes the discovery and service description procedure. After getting the user friend list, the DC traverses this list and check in its own database whether that user already registered to the DLNA service. In the SNS, each user has a unique ID; therefore the DC can easily do that.

For all user friends' ID that registered DLNA service, the DC already has information about their content. Hence, the DC can synthesize all DLNA contents in corresponding home networks and build its own Content Directory Service (CDS) for that user. The root of directory tree is the cloud. The first leaf nodes are named after the HGs. The DC plays a role as a DMS but its content locations point to the corresponding HG. From the view of a DMR, the DC is look like a normal DMS in its home network.

After that, the DC communicates with the $\mathrm{HG}$ to get information about all contents in that home network. Because the HG is a member of the home network, it can send DLNA SEARCH message and get information about all devices in its home network and their contents. The HG also builds its own CDS. The root of directory tree is named after the HG. The first leaf nodes are named after DMSs in its network. All locations are changed to the public IP address of the HG These modifications are stored in a mapping table in the HG database for querying when the $\mathrm{HG}$ receives the request from the DC. The HG advertises this CDS to the DC. Based on this information, the DC builds its own CDS.

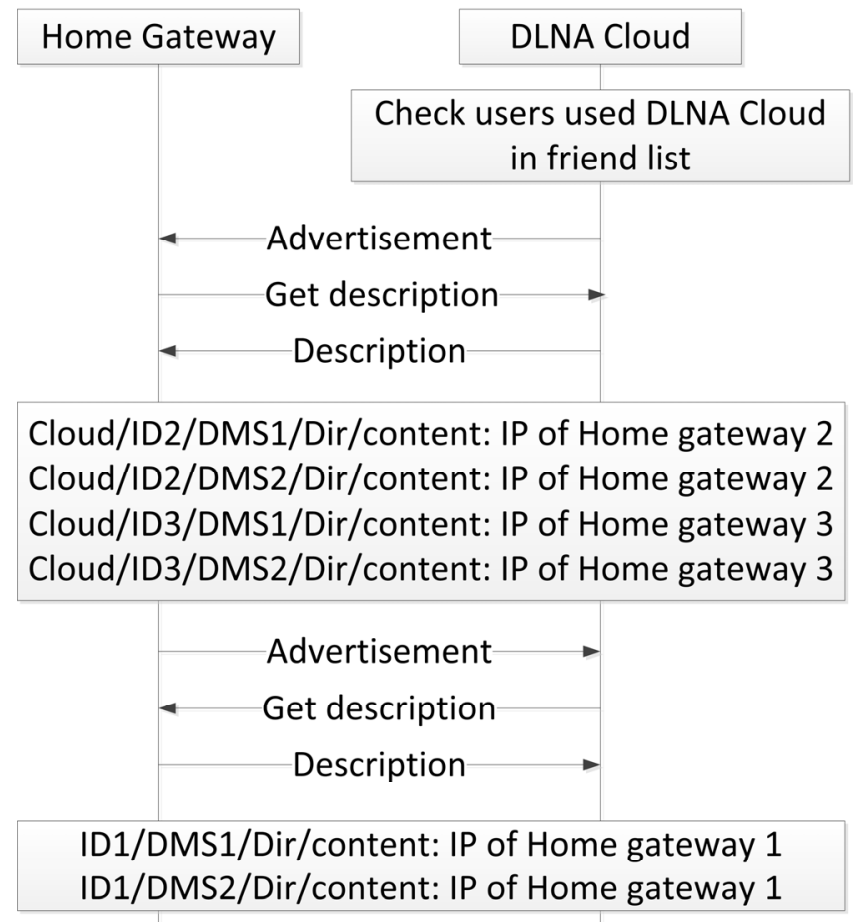

Figure 4. Discovery and service description procedure

\section{3) Media Transference Procedure}

Fig. 5 describes the media transference procedure in the scenario that a user in home network 1 wants to use DMR1 to watch the media content from DMS2 in home network 2.
DMR1 does not know the presence of the DMS1. It receives the advertisement from the HG1 and thinks that the content is located at the HG1. It sends GET_CONTENT message to the HG1. The HG1 in turn checks its own mapping table and knows that content is being located in home network 2. So it translates the IP address and port packet before forwarding this message to the HG2. Similarly, the HG2 checks its mapping table and translates the IP address and the port field in the packets, then send them to DMS1. In turn, DMS1 sends the content back to HG2 in the way that the DLNA device does. In the reserve way, DLNA packets are translated the port and the address before sending to DMR1 in home network 1. Finally, the content is displayed in DMR1 screen. This procedure does not invoke to the DC.

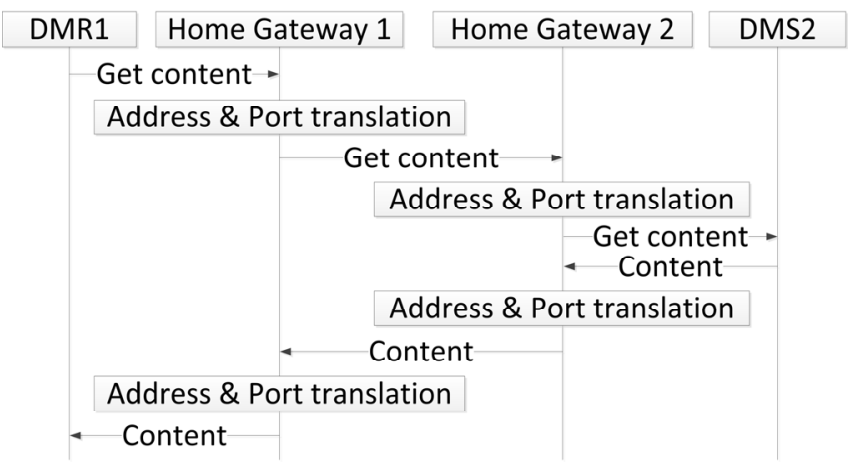

Figure 5. Media transference procedure

\section{4) Update Procedure}

Figure 6 shows the procedure for updating changes in the home network to the DC. When a DLNA device joins or leaves the network, it broadcasts the ALIVE or BYEBYE message in the home network, respectively. The HG discovers these changes by receiving messages from DLNA devices and has the responsibility to notify to the DC. Subsequently, the DC broadcasts the UPDATE message to all HGs that are in relationship with the home network that has changes.

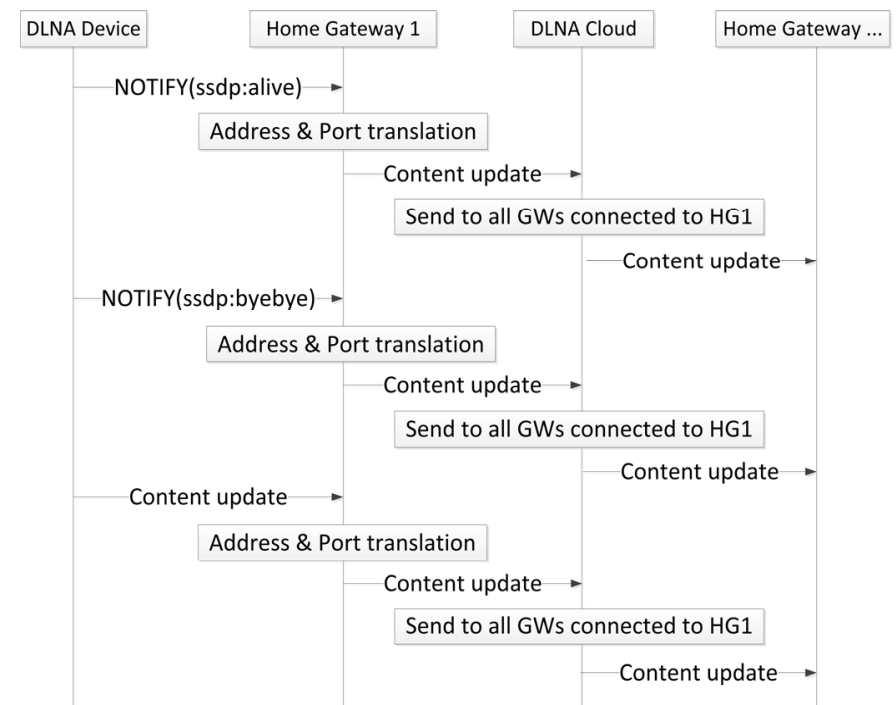

Figure 6. Update procedure 


\section{IV.PERFORMANCE ANALYSIS}

We define the service delay as the time period to complete one procedure between two entities in the system. Accordingly, $D_{1}$ is the service delay of registration and authentication procedure. $D_{2}$ is the service delay of discovery and service description procedure. Similarly, $D_{3}$ and $D_{4}$ is the service delay of media transference and update procedure, respectively. $S_{m}$ is the size of message $m$. Herein, every textbased message is supposed as the command message, messages for exchanging media stream are called data message. $D_{x, y}$ denotes the hop distance between two network entities, $x$ and $y$, and $P_{x}$ denotes the processing cost required by doing task $x$. Assuming that every devices in home network is connected together by wireless links via a access point, these values can be calculated as the sum of the signalling transmission cost and the processing delay cost issued in each entity and are expressed by

$$
\begin{aligned}
& D_{1}=3 \cdot\left(\frac{S_{\text {command }}}{B_{w}}+L_{w}\right)\left(d_{H G, D C}\right) \\
& +4 .\left(\frac{S_{\text {command }}}{B_{w}}+L_{w}\right)\left(d_{D C, S N S}\right) \\
& +2 .\left(\frac{S_{\text {command }}}{B_{w}}+L_{w}\right)\left(d_{H G, S N S}\right) \\
& D_{2}=6 \cdot\left(\frac{S_{\text {command }}}{B_{w}}+L_{w}\right)\left(d_{H G, D C}\right) \\
& D_{3}=\left(\frac{1+q}{1-q}\right)\left(\frac{S_{\text {command }}}{B_{w l}}+L_{w l}\right)\left(d_{D M R 1, H G 1}+d_{H G 2, D M S 2}\right) \\
& +\left(\frac{1+q}{1-q}\right)\left(\frac{S_{d a t a}}{B_{w l}}+L_{w l}\right)\left(d_{D M R 1, H G 1}+d_{H G 2, D M S 2}\right) \\
& +\left(\frac{S_{\text {command }}+S_{\text {data }}}{B_{w}}+2 L_{w}\right)\left(d_{H G 1, H G 2}\right) \\
& +4 P_{\text {port } \& \text { address translation }} \\
& D_{4}=\left(\frac{1+q}{1-q}\right)\left(\frac{S_{\text {command }}}{B_{w l}}+L_{w l}\right)\left(d_{D M S, H G}\right) \\
& +2 \cdot\left(\frac{S_{\text {command }}}{B_{w}}+2 L_{w}\right)\left(d_{H G, D C}\right) \\
& +P_{\text {port }} \& \text { address translation }
\end{aligned}
$$

where $B_{w}$ and $B_{w l}$ are the bandwidth of the wired and wireless links; $L_{w}$ and $L_{w l}$ are the link delay of the wired and wireless links, respectively, and $\mathrm{q}$ is the probability of wireless link failure. Because all signalling procedures are carried out before users want to watch the media, user experience depends a lot on the service delay of media transference procedure which is expressed in (3). With the Internet infrastructure nowadays, the media can be streamed smoothly from one home network to another home network.

\section{Conclusions}

This paper proposes a new architecture allow DLNA networks which originally operate independently can be connected together. Accordingly, DLNA users can easily share multimedia content with their friends over the Internet. The DC plays the key role in this architecture. It manages all HGs and is the intermediate entity for exchanging information between HGs. The SNS is used as the external entity for user authentication and maintaining the relationship and sharing policy. The procedures are proposed for the communication between components in the system. The DLNA HGs in this paper can be implemented as the additional feature of the access point or the set top box in the home network.

\section{ACKNOWLEDGMENT}

This research was supported by the MSIP (Ministry of Science, ICT \& Future Planning), Korea, under the Convergence-ITRC (Convergence Information Technology Research Center) support program (NIPA-2013-H0401-131004) supervised by the NIPA.

\section{REFERENCES}

[1] Digital Living Network Alliance, "DLNA networked device interoperability guidelines," Digital Living Network Alliance, 2006.

[2] J. Kim, Y. Oh, H. Lee, E. Paik, and K. Park, "Implementation of the DLNA Proxy System for Sharing Home Media Contents," IEEE Trans. Consumer Electronics, vol. 53, no. 1, pp. 139-144, Feb. 2007.

[3] Y. Oh; H. Lee; J. Kim; E. Paik; K. Park, "Design of an Extended Architecture for Sharing DLNA Compliant Home Media from Outside the Home," IEEE Trans. Consumer Electronics, vol. 53, no. 2, pp. 542 547, May 2007

[4] D. Diaz-Sanchez, F. Almenarez, A. Marin, D. Proserpio, and P. Arias, "Media cloud: an open cloud computing middleware for conten management," IEEE Transactions on Consumer Electronics, vol. 57, no. 2, pp. 970-978, May 2011.

[5] C. Lai, Y. Huang, and H. Chao, "DLNA-Based Multimedia Sharing System for OSGI Framework with Extension to P2P Network," IEEE System Journal, vol. 4, no.2, pp. 262-270, Jun 2010.

[6] C. Hu; H. Lin; B. Hsieh; Y. Hsu, "Integrated home gateway design for enabling P2P content sharing in UPnP-based home networks," IEEE International Conference on Consumer Electronics (ICCE) 2011, pp. 229-230, 9-12 Jan. 2001.

[7] H. Nam; Y. Kim, "Contents sharing using PPSP in DLNA-based networks," International Conference on Information Networking (ICOIN) 2013, pp. 320-323, 28-30 Jan. 2013.

[8] M. Armbrust, A. Fox, R. Griffith, A. D. Joseph, R. H. Katz, A Konwinski, G. Lee, D. A. Patterson, A. Rabkin, I. Stoica, and M. Zaharia, "Above the Clouds: A Berkeley View of Cloud Computing," EECS Department, University of California, Berkeley UCB/EECS2009-28, Feb. 2009.

[9] W. Tek Tsai, X. Sun, J. Balasooriya, "Service-Oriented Cloud Computing Architecture," Department of Computer Science, Arizona State University, USA, Seventh International Conference on Information Technology, 2010. 